\title{
Form factors and non-local Multiplicative Anomaly for fermions with background torsion
}

\author{
G. de Berredo-Peixoto* and A. Espinosa Maicát \\ Departamento de Física, ICE, Universidade Federal de Juiz de Fora \\ Campus Universitário - Juiz de Fora, MG, Brazil 36036-330
}

\begin{abstract}
We analyse the Multiplicative Anomaly (MA) in the case of quantized massive fermions coupled to a background torsion. The one-loop Effective Action (EA) can be expressed in terms of the logarithm of determinant of the appropriate first-order differential operator acting in the spinors space. Simple algebraic manipulations on determinants must be used in order to apply properly the Schwinger-DeWitt technique, or even the covariant perturbation theory (Barvinsky and Vilkovisky, 1990), which is used in the present work. By this method, we calculate the finite nonlocal quantum corrections, and analyse explicitly the breakdown of those algebraic manipulations on determinants, called by MA. This feature comes from the finite non-local EA, but does not affect the results in the UV limit, in particular the beta-functions. Similar results was also obtained in previous papers but for different external fields (QED and scalar field).
\end{abstract}

Keywords: Fermionic determinants, Multiplicative Anomaly, Effective Action, Torsion, Non-local terms.

PACS: $04.62 .+\mathrm{v} ; 11.15 . \mathrm{Kc} ; 11.10 . \mathrm{Hi}$

\section{Introduction}

The Effective Action (EA) can be considered as an important tool for studying quantum effects in field theories. It should be derived for a given Quantum Field Theory (QFT) taking into

*Email address: guilherme@fisica.ufjf.br

${ }^{\dagger}$ Email address: maica@fisica.ufjf.br 
account its ambiguities: one has to distinguish unambiguous physical effects from the technical features in the calculations.

A relevant ambiguity in QFT concerns, for example, the dependence on the renormalization point, which is described by the parameter $\mu$ in the Minimal Subtraction (MS) scheme adopted in the renormalization procedure. Another ambiguity is the gauge-fixing dependence, which, in principle, can be eliminated on-shell. One can say that many properties obtained in studying the running couplings parameters depend on the renormalization scheme. For example, the renormalization group $\beta$-functions in massive theories calculated in MS scheme are different from the ones calculated using the more physical momentum subtraction scheme. In the low energies regime, the approach of momentum subtraction enables one to observe the decoupling phenomenon, which in QED is known as the Appelquist and Carazzone theorem [1.

There are other ambiguities in quantum contributions, but we can mention an interesting property of the UV divergences: the leading logarithmic divergences define the most stable and universal part of quantum corrections. Let us point out that the UV limit of $\beta$-functions, which is strongly related to UV-divergences, does not depend on the renormalization scheme. On the other side, the calculational techniques, valid for studying the unambiguous UV part (specifically the logarithmic divergences), fails when one investigates the finite non-leading part of one-loop EA, which present then some ambiguity. This kind of ambiguity, coming from what is called the non-local multiplicative anomaly (MA), was treated in recent papers, where different examples was studied: (i) finite 1-loop quantum corrections from massive fermionic fields in electromagnetic background in curved space, Ref. [2]; and (ii) finite 1-loop quantum corrections from massive fermionic fields in Yukawa model (also in curved space), Ref. [3]. It is worth mentioning that this non-local MA is different from what is investigated by many authors in previous papers [4, 5, 6, 7], by using the $\zeta$-regularization. In those works, the MA can be related to the ambiguity of the choice of $\mu$ itself [6, 7]. One can say that the MA which we are considering is a non-local version of earlier MA. In this sense, we are dealing with a different kind of ambiguity of EA.

In this paper, we are going to investigate the MA in the finite 1-loop quantum corrections of massive Dirac fields in the background of an axial vector field, along with gravity. The relevance of this issue is obvious by the great number of research papers on CPT/Lorentz violating theories [8] and also on theories with torsion [9]. In the minimal coupling between fermions and torsion, only the axial component of torsion takes position in the mathematical description (see the recent review [10]), thus an axial background field is useful not only to describe CPT/Lorentz violation, but also to describe torsion issues, ranging from atomic physics to aspects on Quantum Field Theory, Cosmology and Astrophysics. It does not matter if one considers torsion effects on Cosmology or CPT/Lorentz violating theories: in most interesting cases, quantum corrections of quantized matter play relevant role in physical phenomena 1 , such that the MA in finite 1-loop

\footnotetext{
${ }^{1}$ This scenario is not sensitive to the fact that torsion quantization meets serious problems of renormalizability
} 
EA can not be thought as a mathematical feature without physical consequences.

This paper is organized as follows. In Section 2, we describe briefly the relevant aspects of the covariant perturbation theory which are useful for calculating the non-local MA. This calculation is performed in Section 3, for massive fermions coupled to an external axial vector. The results are given explicitly and in Section 4 we deserve some attention to the effects of MA in the beta functions. Finally, in Section 5, we draw our final comments and conclusions.

\section{Preliminary considerations}

Let us consider the quantum corrections coming from free massive fermion fields coupled to the background torsion 2. The action reads

$$
S_{f}=\int d^{4} x \sqrt{-g} \bar{\psi} \hat{H} \psi
$$

where

$$
\hat{H}=i \gamma^{\mu} \nabla_{\mu}+m \hat{1}+\eta \gamma^{5} \gamma^{\mu} S_{\mu}
$$

Here $\hat{1}$ is the identity operator, $S_{\mu}$ is the axial vector, which can be identified with the $b_{\mu}$ parameter in CPT/Lorentz violating theories [8] and also with external torsion, and $\eta$ is the non-minimal coupling parameter (the value $\eta=1 / 8$ describes minimal coupling with torsion).

The one-loop EA can be understood as the classical action of background fields plus the one-loop correction (the subject of our interest) [12]:

$$
\bar{\Gamma}^{(1)}=-\ln \operatorname{Det} \hat{H}
$$

Here and below, we use Euclidean signature. We shall compute the expression (2) through the heat-kernel method and the Schwinger-DeWitt technique (see Refs. [12, 13, 14]), and this requires reducing the problem to the derivation of $\ln$ Det $\hat{\mathcal{O}}$, where the general form for $\hat{\mathcal{O}}$ can be found as

$$
\hat{\mathcal{O}}=\hat{\square}+2 \hat{h}^{\mu} \nabla_{\mu}+\hat{\Pi}
$$

This method of calculation is not directly suited for the first order differential operator $\hat{H}$. However, one can overcome this difficulty by multiplying $\hat{H}$ by another operator $\hat{H}_{1}$ and using the relation

$$
\ln \operatorname{Det} \hat{H}=\ln \operatorname{Det}\left(\hat{H} \hat{H}_{1}\right)-\ln \operatorname{Det} \hat{H}_{1} \text {. }
$$

and unitarity [11].

${ }^{2}$ We mean that they are coupled to an external axial vector. 
The most obvious choice is of course $3 \hat{H}_{1}=\hat{H}$, so that $\ln$ Det $\hat{H}$ is obtained directly by calculation of $(1 / 2) \ln$ Det $(\hat{H} . \hat{H})$. It turns out, however, that this procedure makes computation much more complicated than the one made by an appropriate choice for $\hat{H}_{1}$, say,

$$
\hat{H}_{1}=-i \gamma^{\mu} \nabla_{\mu}+m \hat{1}+\eta \gamma^{5} \gamma^{\mu} S_{\mu}
$$

It is possible to consider also different possibilities, such as

$$
\begin{aligned}
& \hat{H}_{2}=-i \gamma^{\mu} \nabla_{\mu}+m \hat{1}, \\
& \hat{H}_{3}=-i \gamma^{\mu} \nabla_{\mu}-m \hat{1} .
\end{aligned}
$$

The one-loop EA is related to the coincidence limits

$$
\lim _{x \rightarrow x^{\prime}} \hat{a}_{k}\left(x, x^{\prime}\right)=\hat{a}_{k} \mid
$$

which appear in its proper-time expansion (see more details in Ref. [13, 14]) throughout

$$
\bar{\Gamma}^{(1)}=-\frac{1}{2} \operatorname{Tr} \int_{0}^{\infty} \frac{d s}{s} \hat{U}_{0}\left(x, x^{\prime} ; s\right) \sum_{k=0}^{\infty}(i s)^{k} \hat{a}_{k}\left(x, x^{\prime}\right),
$$

where Tr means integration in $x$ of quantities in the limit $x \rightarrow x^{\prime}$ as well as the ordinary trace in discrete labels (i.e., $\operatorname{Tr}=\int d^{4} x \lim _{x \rightarrow x^{\prime}} \operatorname{tr}$ ), the minus sign is present because of the Grassmann parity (we consider here only the fermionic sector), and $\hat{U}_{0}\left(x, x^{\prime} ; s\right)$ is an operator function related to the geodesic distance between $x$ and $x^{\prime}$ and the Van-Vleck-Morette determinant.

The coefficients $\hat{a}_{k} \mid$ are useful to describe the EA in several field theory models. For instance, in the two-dimensional spacetime, $\hat{a}_{1} \mid$ defines logarithmic divergences. In four dimensions, $\hat{a}_{1} \mid$ describes quadratic divergences, while the logarithmic ones are given by $\hat{a}_{2} \mid$ (the same happens with $\hat{a}_{3} \mid$ in six dimensions, and so on so forth). In four dimensions, all summation with $k>2$ represent finite contributions in the EA.

Fortunately, we do not need to calculate all the coefficients to investigate the MA from finite contributions, because Barvinsky and Vilkovisky [15] and also Avramidi [16] obtained an equivalent EA but expressed as a different summation, in powers of curvature (i.e., in powers of quantities with dimension of curvature). Each coefficient of this new expansion (called form factors) has all non-localities coming from the finite part. In Ref. [17], this method was used to calculate the complete form factors and $\beta$-functions for some massive fields at 1-loop level (See also Refs. [18, 19] for similar calculations). The approach developed in Refs. [15, 16] is sometimes called covariant perturbation theory.

\footnotetext{
${ }^{3}$ For practical purposes and calculational convenience, this is realized by considering $\hat{H}_{1}=-\hat{H}$. The difference will be just a constant term which does not change the main results.
} 
The Schwinger-DeWitt algorithm enables one to obtain $\hat{a}_{1} \mid$ and $\hat{a}_{2} \mid$ for the differential operator in the general form (3):

$$
\begin{gathered}
\hat{a}_{1} \mid=\hat{P}=\hat{\Pi}+\frac{\hat{1}}{6} R-\nabla_{\mu} \hat{h}^{\mu}-\hat{h}_{\mu} \hat{h}^{\mu}, \\
\hat{a}_{2} \mid=\frac{\hat{1}}{180}\left(R_{\mu \nu \alpha \beta}^{2}-R_{\mu \nu}^{2}+\square R\right)+\frac{1}{2} \hat{P}^{2}+\frac{1}{6}(\square \hat{P})+\frac{1}{12} \hat{S}_{\mu \nu}^{2},
\end{gathered}
$$

where we denote $R_{\mu \nu \alpha \beta}^{2}=R_{\mu \nu \alpha \beta} R^{\mu \nu \alpha \beta}$ (and so on) and

$$
\hat{S}_{\mu \nu}=\hat{1}\left[\nabla_{\mu}, \nabla_{\nu}\right]+\nabla_{\mu} \hat{h}_{\nu}-\nabla_{\nu} \hat{h}_{\mu}+\hat{h}_{\mu} \hat{h}_{\nu}-\hat{h}_{\nu} \hat{h}_{\mu}
$$

\section{Calculation of the one-loop form factors}

Using the technique of covariant perturbation theory, one can express the one-loop EA (9) in the form of an expansion in powers of fields strengths (or curvatures). Up to second order in curvatures, it can be written as

$$
\begin{aligned}
\bar{\Gamma}^{(1)}= & -\frac{1}{2} \int_{0}^{\infty} \frac{d s}{s} \frac{\mu^{4-2 \omega}}{(4 \pi s)^{\omega}} \int d^{2 \omega} x \sqrt{g} e^{-s m^{2}} \operatorname{tr}\left\{\hat{1}+s \hat{P}+s^{2}\left[R_{\mu \nu} f_{1}(\tau) R^{\mu \nu}\right.\right. \\
& \left.\left.+R f_{2}(\tau) R+\hat{P} f_{3}(\tau) R+\hat{P} f_{4}(\tau) \hat{P}+\hat{S}_{\mu \nu} f_{5}(\tau) \hat{S}^{\mu \nu}\right]\right\}
\end{aligned}
$$

where $\omega$ is the dimensional regularization parameter, $\mu$ is the renormalization parameter with dimension of mass and the functions $f_{i}$ of $\tau=-s \square$ are given by

$$
\begin{aligned}
& f_{1}(\tau)=\frac{f(\tau)-1+\tau / 6}{\tau^{2}} \\
& f_{2}(\tau)=\frac{f(\tau)}{288}+\frac{f(\tau)-1}{24 \tau}-\frac{f(\tau)-1+\tau / 6}{8 \tau^{2}} \\
& f_{3}(\tau)=\frac{f(\tau)}{12}+\frac{f(\tau)-1}{2 \tau} \\
& f_{4}(\tau)=\frac{f(\tau)}{2} \\
& f_{5}(\tau)=\frac{1-f(\tau)}{2 \tau}
\end{aligned}
$$

with

$$
f(\tau)=\int_{0}^{1} d \alpha e^{-\alpha(1-\alpha) \tau} .
$$

In order to deal with the integrations in parameter $s$, we indicate the works in Ref. [17], where the reader can find more technical details. Let us consider only the terms containing the $S_{\mu}$ field, thus we disregard the vacuum contributions (for example, $R f_{2}(\tau) R$ ) as well as the total 
derivative terms. Then, we keep attention only to form factors containing $f_{3}, f_{4}$ and $f_{5}$. After cumbersome calculation, one can perform some tricky integrations and rearrange the relevant terms of (12) to get the following result, for Euclidean EA in 4 dimensions:

$$
\begin{aligned}
\bar{\Gamma}^{(1)}= & -\frac{1}{2(4 \pi)^{2}} \int d^{4} x \sqrt{g} \operatorname{tr}\left\{\hat{P}\left(A+\frac{1}{2 \epsilon}\right) \hat{P}+\hat{P}\left(\left(a^{2}-4\right) \frac{A}{6 a^{2}}-\frac{1}{18}\right) R\right. \\
& \left.+\hat{S}_{\mu \nu}\left(\frac{1}{18}+\frac{2 A}{3 a^{2}}+\frac{1}{12 \epsilon}\right) \hat{S}^{\mu \nu}\right\},
\end{aligned}
$$

where

$$
A=1+\frac{1}{a} \ln \left(\frac{2-a}{2+a}\right) ; \quad a^{2}=\frac{4 u}{u+4} ; \quad u=-\square / m^{2},
$$

and the small parameter $\epsilon$ was arbitrarily chosen in terms of $\omega$ according to

$$
-\frac{1}{\epsilon}=\frac{1}{\omega-2}+\gamma+\ln \left(\frac{m^{2}}{4 \pi \mu^{2}}\right),
$$

with $\gamma$ denoting the Euler constant. Notice that the coefficient of $\operatorname{tr}(\hat{P} R)$ has no divergent part $(\propto 1 / \epsilon)$.

\subsection{Universality of 1-loop logarithm divergences}

We shall indicate that the MA does not affect the 1-loop divergences. In order to do so, let us compare, for example, the two quantities

$$
\left.\operatorname{Tr} \ln (\hat{H} \hat{H})\right|_{\text {div }} \quad \text { and }\left.\quad \operatorname{Tr} \ln \left(\hat{H} \hat{H}_{1}\right)\right|_{\text {div }},
$$

where $\hat{H}$ and $\hat{H}_{1}$ are given by Eqs. (11) and (5), respectively. Disregarding the vacuum and superficial contributions, one can calculate $\operatorname{Tr} \hat{a}_{2}$, following the Schwinger-DeWitt technique, and obtain, in $n$ dimensions,

$$
\begin{aligned}
\left(\operatorname{Tr} \hat{a}_{2} \mid\right)_{\hat{H} \hat{H}}= & \int d^{n} x \sqrt{g} 2^{[n / 2]}\left\{\frac{n-2}{12} \eta^{2} S_{\mu \nu}^{2}-(n-2)(n-3) \eta^{2} m^{2} S^{2}\right. \\
& \left.+\frac{n-4}{6}\left(\eta^{2}\left(\nabla_{\mu} S^{\mu}\right)^{2}-\eta^{2} R_{\mu \nu} S^{\mu} S^{\nu}+\frac{1}{2} \eta^{2} R S^{2}+(n-2) \eta^{4} S^{4}\right)\right\}, \\
\left(\operatorname{Tr} \hat{a}_{2} \mid\right)_{\hat{H} \hat{H}_{1}}= & \int d^{n} x \sqrt{g} 2^{[n / 2]}\left\{-2 \eta^{2} m^{2} S^{2}+\frac{1}{6} \eta^{2} S_{\mu \nu}^{2}\right\},
\end{aligned}
$$

where $[n / 2]$ means the integer part of $n / 2, S_{\mu \nu}^{2}=S_{\mu \nu} S^{\mu \nu}, S^{2}=S_{\mu} S^{\mu}$ and $S^{4}=\left(S_{\mu} S^{\mu}\right)^{2}$. The above two expressions coincide only in four dimensions (when $\hat{a}_{2}$ describes logarithm divergences). The same has to happen with $\hat{a}_{1}$ in two dimensions, corresponding to logarithm divergences. Indeed, this is so:

$$
\begin{aligned}
\left(\operatorname{Tr} \hat{a}_{1} \mid\right)_{\hat{H} \hat{H}} & =\int d^{n} x \sqrt{g} 2^{[n / 2]}(2-n) \eta^{2} S^{2}+\text { vacuum }, \\
\left(\operatorname{Tr} \hat{a}_{1} \mid\right)_{\hat{H} \hat{H}_{1}} & =\text { vacuum } .
\end{aligned}
$$

One could verify this mechanism also for $\operatorname{Tr} \hat{a}_{3} \mid$, but this issue has already been discussed for other fields in previous papers [2, 3]. 


\subsection{Multiplicative Anomaly in four dimensions}

Now we proceed with the calculation of form factors from Eq. (14) for four different operators: $\hat{H} \hat{H}, \hat{H} \hat{H}_{1}, \hat{H} \hat{H}_{2}$ and $\hat{H} \hat{H}_{3}$. We consider the four dimensional case and omit the the total derivatives and vacuum terms. By straightforward computation, we achieve

$$
\begin{aligned}
-\frac{1}{2} \operatorname{Tr} \ln (\hat{H} \hat{H})= & -\frac{1}{2(4 \pi)^{2}} \int d^{4} x \sqrt{g}\left\{\eta^{2}\left(\nabla_{\mu} S^{\mu}\right)\left[k_{\nabla S}(a)\right]\left(\nabla_{\nu} S^{\nu}\right)+\right. \\
& +\eta^{2} S_{\mu \nu}\left[k_{S S}(a)+\frac{2}{3 \epsilon}\right] S^{\mu \nu}+\eta^{2} m^{2} S_{\mu}\left[k_{S}(a)-\frac{8}{\epsilon}\right] S^{\mu}+ \\
& \left.+\eta^{4} S^{2}\left[k_{S 4}(a)\right] S^{2}\right\} \\
-\frac{1}{2} \operatorname{Tr} \ln \left(\hat{H} \hat{H}_{1}\right)=- & \frac{1}{2(4 \pi)^{2}} \int d^{4} x \sqrt{g}\left\{\eta^{2} S_{\mu \nu}\left[k_{S S}^{(1)}(a)+\frac{2}{3 \epsilon}\right] S^{\mu \nu}\right. \\
& \left.+\eta^{2} m^{2} S_{\mu}\left[k_{S}^{(1)}(a)-\frac{8}{\epsilon}\right] S^{\mu}\right\} \\
-\operatorname{Tr} \ln \left(\hat{H} \hat{H}_{2}\right)= & -\frac{1}{2(4 \pi)^{2}} \int d^{4} x \sqrt{g}\left\{\eta^{2}\left(\nabla_{\mu} S^{\mu}\right)\left[k_{\nabla S}^{(2)}(a)\right]\left(\nabla_{\nu} S^{\nu}\right)\right. \\
& +\eta^{2} S_{\mu \nu}\left[k_{S S}^{(2)}(a)+\frac{2}{3 \epsilon}\right] S^{\mu \nu}+\eta^{4} S^{2}\left[k_{S 4}^{(2)}(a)\right] S^{2} \\
& \left.+\eta^{2} m^{2} S_{\mu}\left[k_{S}^{(2)}(a)-\frac{8}{\epsilon}\right] S^{\mu}\right\} \\
-\operatorname{Tr} \ln \left(\hat{H} \hat{H}_{3}\right)= & \frac{1}{2(4 \pi)^{2}} \int d^{4} x \sqrt{g}\left\{\eta^{2}\left(\nabla_{\mu} S^{\mu}\right)\left[k_{\nabla S}^{(3)}(a)\right]\left(\nabla_{\nu} S^{\nu}\right)\right. \\
& +\eta^{2} S_{\mu \nu}\left[k_{S S}^{(3)}(a)+\frac{2}{3 \epsilon}\right] S^{\mu \nu}+\eta^{4} S^{2}\left[k_{S 4}^{(3)}(a)\right] S^{2} \\
& \left.+\eta^{2} m^{2} S_{\mu}\left[k_{S}^{(3)}(a)-\frac{8}{\epsilon}\right] S^{\mu}\right\}
\end{aligned}
$$

where the form factors are, for the first scheme,

$$
\begin{aligned}
k_{\nabla S}(a) & =\frac{16 A}{a^{2}}-4 A+\frac{4}{3} \\
k_{S S}(a) & =\frac{16 A}{3 a^{2}}+\frac{4}{9} \\
k_{S}(a) & =\frac{384 A}{a^{2}}-112 A+32 \\
k_{S 4}(a) & =16 A-\frac{64 A}{a^{2}}-\frac{16}{3}
\end{aligned}
$$

for the second scheme,

$$
\begin{aligned}
& k_{S S}^{(1)}(a)=2 A-\frac{8 A}{3 a^{2}}-\frac{2}{9} \\
& k_{S}^{(1)}(a)=-16 A
\end{aligned}
$$


for the third scheme,

$$
\begin{aligned}
k_{\nabla S}^{(2)}(a) & =\frac{8 A}{a^{2}}-2 A+\frac{2}{3} \\
k_{S S}^{(2)}(a) & =A+\frac{4 A}{3 a^{2}}+\frac{1}{9} \\
k_{S 4}^{(2)}(a) & =2 A-\frac{8 A}{a^{2}}-\frac{2}{3} \\
k_{S}^{(2)}(a) & =k_{S}^{(1)}(a),
\end{aligned}
$$

and finally for the fourth scheme,

$$
\begin{aligned}
k_{\nabla S}^{(3)}(a) & =k_{\nabla S}^{(2)}(a) \\
k_{S S}^{(3)}(a) & =k_{S S}^{(2)}(a) \\
k_{S 4}^{(3)}(a) & =k_{S 4}^{(2)}(a) \\
k_{S}^{(3)}(a) & =\frac{160 A}{a^{2}}-56 A+\frac{40}{3} .
\end{aligned}
$$

By Eq. (19), we can obtain the expression for $-\operatorname{Tr} \ln (\hat{H})=-(1 / 2) \operatorname{Tr} \ln (\hat{H} \hat{H})$ : it coincides thus with the expression in the right hand side of Eq. (19). Now that we have

$$
\operatorname{Tr} \ln (\hat{H})=\frac{1}{2} \operatorname{Tr} \ln (\hat{H} \hat{H}),
$$

we obtain easily $\operatorname{Tr} \ln \left(\hat{H}_{1}\right)$ by doing $m \rightarrow-m$ and $S_{\mu} \rightarrow-S_{\mu}$ in (37), what leaves the result unchanged, of course (it has only even powers on $m$ and $\left.S_{\mu}\right)$. Thus, $\operatorname{Tr} \ln \left(\hat{H}_{1}\right)=\operatorname{Tr} \ln (\hat{H})$.

Nevertheless, one can obtain $\operatorname{Tr} \ln \left(\hat{H}_{1}\right)$ by substituting the result (20) into

$$
\operatorname{Tr} \ln \left(\hat{H}_{1}\right)=\operatorname{Tr} \ln \left(\hat{H} \hat{H}_{1}\right)-\operatorname{Tr} \ln (\hat{H})
$$

what should give the exact expression for $\operatorname{Tr} \ln (\hat{H})$, but instead it gives

$$
\begin{aligned}
-\operatorname{Tr} \ln \left(\hat{H}_{1}\right)= & -\frac{1}{2(4 \pi)^{2}} \int d^{4} x \sqrt{g}\left\{\eta^{2}\left(\nabla_{\mu} S^{\mu}\right)\left[k_{\nabla S}^{H_{1}}(a)\right]\left(\nabla_{\nu} S^{\nu}\right)+\right. \\
& +\eta^{2} S_{\mu \nu}\left[k_{S S}^{H_{1}}(a)+\frac{2}{3 \epsilon}\right] S^{\mu \nu}+\eta^{2} m^{2} S_{\mu}\left[k_{S}^{H_{1}}(a)-\frac{8}{\epsilon}\right] S^{\mu}+ \\
& \left.+\eta^{4} S^{2}\left[k_{S 4}^{H_{1}}(a)\right] S^{2}\right\},
\end{aligned}
$$

with

$$
\begin{gathered}
k_{\nabla S}^{H_{1}}(a)=-k_{\nabla S}(a) ; \quad k_{S 4}^{H_{1}}(a)=-k_{S 4}(a), \\
k_{S S}^{H_{1}}(a)=-\frac{32 A}{3 a^{2}}+4 A-\frac{8}{9}, \\
k_{S}^{H_{1}}(a)=-\frac{384 A}{a^{2}}+80 A-32 .
\end{gathered}
$$


The last result is a definitive indication that relation (38) does not hold, and this is precisely what we call MA. One can ask of course if relation $\operatorname{Tr} \ln (\hat{H})=(1 / 2) \operatorname{Tr} \ln (\hat{H} \hat{H})$ itself holds: to detect MA, we must assume it holds, but the contrary hypothesis would mean that MA is present anyway. The analogous feature in calculating $\operatorname{Tr} \ln \left(\hat{H}_{2}\right)$ and $\operatorname{Tr} \ln \left(\hat{H}_{3}\right)$ can also be shown as follows.

Let us notice that just by visual inspection, the expressions for $\operatorname{Tr} \ln \left(\hat{H}_{2}\right)$ and $\operatorname{Tr} \ln \left(\hat{H}_{3}\right)$ can be obtained from $\operatorname{Tr} \ln (\hat{H})$ by the procedure $S_{\mu} \rightarrow 0$. Then,

$$
\operatorname{Tr} \ln \left(\hat{H}_{2}\right)=\operatorname{Tr} \ln \left(\hat{H}_{3}\right)=\text { vacuum and total derivatives, }
$$

so the expressions for $\operatorname{Tr} \ln \left(\hat{H} \hat{H}_{2,3}\right)$ should be both equal to $\operatorname{Tr} \ln (\hat{H})$ in (37) and (19), but they are different as shown by relations (21) and (22).

To summarize what we have so far: the main object of interest is $\operatorname{Tr} \ln (\hat{H})$, which can be calculated by four methods, producing different results, all affected by MA. In the first method, we used $\operatorname{Tr} \ln (\hat{H})=(1 / 2) \operatorname{Tr} \ln (\hat{H} \hat{H})$ and got (19). In the second method, we used (38) and got (39). Finally, in the third and fourth methods we got respectively expressions (21) and (22) for $-\operatorname{Tr} \ln (\hat{H})$. We shall see that the MA is indeed a feature in the IR and intermediate regimes, but suppressed in the UV, in considering the same form factors calculated in this section in the renormalization group equations approach.

\section{Renormalization group and beta-functions}

In this section, we describe the effect of MA in the renormalization of running parameters and show that the beta-functions confirm the generalized version of Appelquist and Carazzone decoupling theorem [1]. We shall consider the calculation of beta-functions in the mass dependent scheme 4 (in contrast to Minimal Substraction Scheme). From the form factor (polarization operator), we subtract the counterterm at the momentum $p^{2}=M^{2}$, with $M$ being the renormalization point.

In order to obtain the beta-function for the effective coupling $\eta$, we apply then the operator

$$
-\lim _{n \rightarrow 4} p \frac{d}{d p}=\lim _{n \rightarrow 4} \frac{4-a^{2}}{4} a \frac{d}{d a}
$$

to the form factor $k_{S S}(a)$, where we have used $u=p^{2} / m^{2}=4 a^{2} /\left(4-a^{2}\right)$. Let us show the result of this calculation for the first method as described in the previous section. We achieve thus the 1-loop beta-function

$$
\beta_{1 \eta}=\frac{\eta^{2}}{(4 \pi)^{2}}\left\{\frac{12-2 a^{2}}{3 a^{2}}+\frac{4-a^{2}}{a^{3}} \ln \left(\frac{2-a}{2+a}\right)\right\}
$$

\footnotetext{
${ }^{4}$ See, for example, [20, 21].
} 
while in the second method we get

$$
\beta_{1 \eta}^{(1)}=\frac{\eta^{2}}{(4 \pi)^{2}}\left\{\frac{7 a^{2}-24}{3 a^{2}}-\frac{a^{4}-12 a^{2}+32}{4 a^{3}} \ln \left(\frac{2-a}{2+a}\right)\right\} .
$$

The above results are quite discrepant, but they provide the same UV limit, which is computed directly by calculating the limit for $a \rightarrow 2$. The result following the third and fourth methods are identical because both form factors related to $S_{\mu \nu}^{2}$-term are identical. We have then

$$
\beta_{1 \eta}^{(2,3)}=\frac{\eta^{2}}{(4 \pi)^{2}}\left\{\frac{a^{2}+12}{12 a^{2}}-\frac{a^{4}-16}{16 a^{3}} \ln \left(\frac{2-a}{2+a}\right)\right\} .
$$

Despite the discrepancy between these three results, in the UV regime we find the same betafunctions (which coincides with the beta-function in Minimal Subtraction Scheme). We obtain

$$
\beta_{1 \eta U V}=\beta_{1 \eta U V}^{(1)}=\beta_{1 \eta U V}^{(2,3)}=\frac{2}{3} \frac{\eta^{2}}{(4 \pi)^{2}} .
$$

We should mention, however, that in the IR limit (i.e., $a \rightarrow 0$ ), the beta-functions describe in all cases the Appelquist and Carazzone decoupling theorem with different behaviors:

$$
\begin{aligned}
& \beta_{1 \eta I R}=\frac{1}{15} \frac{\eta^{2}}{(4 \pi)^{2}} \frac{p^{2}}{m^{2}}+\mathcal{O}\left(\frac{p^{4}}{m^{4}}\right), \\
& \beta_{1 \eta I R}^{(1)}=\frac{1}{5} \frac{\eta^{2}}{(4 \pi)^{2}} \frac{p^{2}}{m^{2}}+\mathcal{O}\left(\frac{p^{4}}{m^{4}}\right), \\
& \beta_{1 \eta I R}^{(2,3)}=\frac{1}{10} \frac{\eta^{2}}{(4 \pi)^{2}} \frac{p^{2}}{m^{2}}+\mathcal{O}\left(\frac{p^{4}}{m^{4}}\right) .
\end{aligned}
$$

In above formulas, the results are expressed in terms of the squared external momentum $p^{2}$ and the fermion mass $m$. Notice that the most dominant terms are proportional to $p^{2} / m^{2}$ which is already very small in the IR limit $\left(p^{2}<<m^{2}\right)$. So, we conclude that the Appelquist and Carazzone theorem definitely holds in the case of torsion, but the coefficients of $\mathcal{O}\left(\frac{p^{2}}{m^{2}}\right)$ depends on the calculational scheme. This situation is pretty much the same as for QED [2] and Yukawa model [3].

Nevertheless, one can find an intriguing feature in our results, in particular on the expressions for the form factors $\left(\nabla_{\mu} S^{\mu}\right)\left[k_{\nabla S}(a)\right]\left(\nabla_{\nu} S^{\nu}\right)$ and $S^{2}\left[k_{S 4}(a)\right] S^{2}$. As a confirmation of known results (see, e.g., Ref. [11]), there are no one-loop divergent terms $\sim\left(\nabla_{\mu} S^{\mu}\right)^{2}$ and $\sim\left(S_{\mu} S^{\mu}\right)^{2}$; although we find here the appearance of finite non-local corrections.

That is a very unusual feature in considering the issue of the decoupling mechanism. For instance, there are no such beta-functions in MS scheme corresponding to these interactions, indeed in the UV limit the application of $p \frac{d}{d p}$ to $k_{\nabla S}(a)$ and $k_{S 4}(a)$ vanishes in both cases and in all schemes. However, in intermediate and IR regimes, the beta-functions are non-trivial (and in fact are sensitive to calculational scheme, or MA). Thus, in these cases the word decoupling is not clear (because not only in the IR, the beta-functions go to zero as $\mathcal{O}\left(\frac{p^{2}}{m^{2}}\right)$, but also in UV they go to zero). 
One should mention that, in Ref. [11, it was shown that the spin-0 related term $\left(\nabla_{\mu} S^{\mu}\right)^{2}$ did not appear in the one-loop level, in a very different scenario where fermions and massive dynamical torsion are all quantized, but appeared in the two-loop level thanks to some specific form of the one-loop torsion quantum effects. In the present work, we get the result that this renormalizability and unitarity breaking term, $\left(\nabla_{\mu} S^{\mu}\right)^{2}$, manifests already in one-loop level.

\section{Conclusions and final remarks}

We have confirmed real inconsistency in the formula $\operatorname{Tr} \ln \left(\hat{H}_{1} \hat{H}_{2}\right)=\operatorname{Tr} \ln \left(\hat{H}_{1}\right)+\operatorname{Tr} \ln \left(\hat{H}_{2}\right)$ when applied to massive fermions coupled with an external torsion field. This feature was presented for other cases in previous papers [2, 3]. The ambiguity, called non-local Multiplicative Anomaly (MA), is supressed in calculation of Schwinger-DeWitt coefficient $a_{2}$, in four dimensions, which corresponds to local 1-loop logarithmic divergences.

The UV regime is not sensitive to MA, in contrast to what happens in other regimes, specially in the IR. It is typically a feature coming from the finite non-local part of quantum corrections, as described by the expansion of $\operatorname{Tr} \ln (\hat{H})$ in powers of curvature. It turns out that the betafunctions are affected by MA in the IR and intermediate regimes, but are universal in the UV. We found also finite non-local contribution to some beta-functions which are absent in UV limit, a feature which deserves further attention.

It is interesting that this universality in the UV limit could be derived directly from the finite non-local 1-loop effective action, in the same time as we know that the universality of beta-functions in the UV comes from the usual consideration of local logarithmic divergences, which are unambiguous. Thus, in our opinion, this is an indication that all possible ambiguity plaguing the results comes from the MA.

\section{Acknowledgements}

G.B.P. is grateful to CNPq and FAPEMIG for partial support. A.E.M. is grateful to CAPES

for the PhD support program. The authors acknowledge I.L. Shapiro for suggesting to study the MA for fermions in external torsion, as well as reading the manuscript.

\section{References}

[1] T. Appelquist and J. Carazzone, Phys. Rev. 11 (1975) 2856.

[2] B. Gonçalves, G. de Berredo-Peixoto and I.L. Shapiro, Phys. Rev. D 80 (2009) 104013; Int. J. Mod. Phys. A25 (2010) 2382-2390.

[3] G. de Berredo-Peixoto, D.D. Pereira and I.L. Shapiro, Phys. Rev. D 85 (2012) 064025. 
[4] E. Elizalde, L. Vanzo and S. Zerbini, Commun. Math. Phys. 194 (1998) 613;

G. Cognola, E. Elizalde and S. Zerbini, Commun. Math. Phys. 237 (2003) 507, hep-th/9910038;

E. Elizalde, G. Cognola and S. Zerbini, Nucl. Phys. B532 (1998) 407;

E. Elizalde and M. Tierz, J. Math. Phys. 45 (2004) 1168, hep-th/0402186.

[5] T.S. Evans, Phys. Lett. B 457 (1999) 127;

[6] J.S. Dowker, On the relevance of the multiplicative anomaly, hep-th/9803200;

[7] J.J. McKenzie-Smith and D.J. Toms, Phys. Rev. D 58 (1998) 105001.

[8] D. Colladay and V.A. Kostelecky, Phys. Rev. D55 (1997) 6760-6774; Phys. Rev. D58 (1998) 116002.

[9] F.W. Hehl, P. von der Heyde, G.D. Kerlick, and J.M. Nester, Rev. Mod. Phys. 48 (1976) 393-416.

[10] I.L. Shapiro, Phys. Rept. 357 (2002) 113.

[11] G. de Berredo-Peixoto, J.A. Helayël-Neto and I.L. Shapiro, JHEP 02 (2000) 003.

[12] I.L. Buchbinder, S.D. Odintsov and I.L. Shapiro, Effective Action in Quantum Gravity, Institute of Physics, Bristol, 1992.

[13] Bryce S. DeWitt, Dynamical Theory of Groups and Fields, Gordon and Breach, New York, 1965 .

[14] I.G. Avramidi, Covariant methods for the calculation of the effective action in quantum field theory and investigation of higher-derivative quantum gravity. ( $\mathrm{PhD}$ thesis, Moscow University, 1986); hep-th/9510140.

[15] A.O. Barvinsky and G.A. Vilkovisky, Nucl. Phys. B333 (1990) 471.

[16] I. G. Avramidi, Yad. Fiz. (Sov. Journ. Nucl. Phys.) 49 (1989) 1185.

[17] E.V. Gorbar and I.L. Shapiro, JHEP 02 (2003) 021, hep-ph/0210388; JHEP 06 (2003) 004, hep-ph/0303124]; G. de Berredo-Peixoto, E.V. Gorbar and I.L. Shapiro, Class. Quantum Grav. 21 (2004) 2281.

[18] K.A. Milton, Phys. Rev. D 15 (1977) 2149.

[19] Yu.V. Gusev and A.I. Zelnikov, Phys. Rev. D 59 (1999) 024002; e-Print: hep-th/9807038.

[20] P. Ramond, Field Theory: a Modern Primer, Westview, Boulder, 2001.

[21] A.V. Manohar, arXiv:hep-ph/9606222. 